EDITORIAL

Rev Colombiana Cienc Anim 2016; 8(Supl):258-259.

\title{
Publicaciones científicas universitarias
}

\author{
University scientific publications
}

PERIÑAN-PETRO, VICENTE, ${ }^{1}$ M.Sc.

${ }^{1}$ Rector Universidad de Sucre, Colombia. Correspondencia: vicente.perinan@unisucre.edu.co

La edición de este suplemento de la Revista Colombiana de Ciencia Animal - RECIA, publicación científica de la Facultad de Ciencias Agropecuarias de la Universidad de Sucre, en donde se compilan los temas tratados en el Primer Seminario sobre Etología y Bienestar Animal, recientemente llevado a cabo en nuestro claustro, escenario en el cual se expusieron y discutieron los diversos aspectos que la temática en mención convoca y en donde docentes investigadores del programa de Zootecnia, de Instituciones nacionales y extrajeras participaron, nos permite entregar a la comunidad académica a manera de escritos científicos los resultados de este evento y otras producciones investigativas que de forma complementaria muestran el avance que en este sentido se está logrando.

Como lo señala AGUIRRE-ROMERO (1977), uno de los elementos clave en la vida académica, en lo referente a los docentes e investigadores, es la publicación de los resultados de su trabajo. Se puede decir sin demasiadas reservas que una gran parte de la vida académica está condicionada por la publicación. No en vano, los profesores son evaluados conforme a su volumen de publicación, las que son fundamentales para su desarrollo y promoción. Sin embargo, hay que notar entre otros aspectos y con acogimiento a este mismo autor, que la capacidad de investigación de una universidad siempre está por encima de su capacidad de publicación; igualmente es justo señalar, que la capacidad de publicación no significa necesariamente capacidad de difusión.

Pero la labor del académico, que debe gravitar entre la investigación y la docencia, conformando una unidad de acción es el cimiento y quehacer real del investigador; de ésta forma le aporta al estudiante contenidos que eleven su nivel académico; esta unidad permite al profesor reflexionar sobre sus inquietudes intelectuales y científicas en la medida en que investiga y traspasa parte de esas inquietudes y conocimientos a un auditorio preparado; de esta manera logra acercar al estudiante realmente a la realidad, con conocimientos extraídos de ella misma y superando el nivel mediocre y pragmatista (VÉLEZ y DÁVILA, 1984;

Más allá de los conceptuado anteriormente y como parte de los objetivos misionales de la Universidad, no se debe dejar de lado la extensión, ella en si misma también hace valioso aporte al desarrollo creativo y a la investigación, En palabras de CHACÍN et al. (2007), la extensión universitaria representa una posibilidad real para la generación de nuevos conocimientos al enriquecer las actividades de docentes y estudiantes, permitiéndoles abordar de manera más integral y eficiente los problemas, poniendo en evidencia nuevos ámbitos de trabajo e investigación al develar nuevas necesidades, carencias y fortalezas de la realidad en la cual se inserta, permitiendo validar en el campo los resultados tanto de la experiencia docente como de la acción investigativa. 
Por lo tanto, la labor investigativa como concepción general de la universidad, va más allá de ser un simple sustantivo, hace parte de la sinergia que identifica la academia. Las publicaciones que de ella se derivan son el sustento universal de la labor que sus docentes acometen para comprender mejor la realidad y llevar por el camino de la excelencia a quienes como sus alumnos hacen parte del sendero a recorrer. La Universidad como un todo, debe obligatoriamente cada día reconstruirse sobre la base de sus propios descubrimientos, como parte de un proceso continuo e inagotable que subyace en la investigación y en la divulgación de sus resultados.

\section{Referencias}

AGUIRRE-ROMERO, J. 1977. Las revistas digitales y la vida académica. Disponible en: (http:// pendientedemigracion.ucm.es/info/multidoc/multidoc/revista/cuad6-7/aguirre.htm\#bio. Consultada: 20-11-2016.

CHACÍN, B.; GONZÁLEZ, M.; TORRES, Y. 2007. Crítica a la generación del conocimiento en la extensión universitaria: aproximación a un protocolo de investigación innovativa. Universidad Pedagógica Experimental Libertador, Venezuela. Laurus 13 (24):215-240.

VÉLEZ, P.I.; DÁVILA L.R. 1984. De la investigación universitaria en Colombia. Educación Superior y Desarrollo 3 (1):48-54. 